Free Communications

Contr. Gynec. Obstet., vol. 16, pp. 313-321 (Karger, Basel 1987)

A Survey amongst 800 Members of the Endometriosis

Society

Caroline Hawkridge

Endometriosis Society, London, England

The Endometriosis Society is a self-help organisation for endometriosis sufferers in the UK. It is also concerned with promoting further research and has conducted a survey amongst 800 members.

The questionnaire covered gynaecological history, diagnosis of endometriosis and its treatment. Women were also asked how they found out more about the disorder and their beliefs about its cause.

Findings of particular interest are already emerging. For example, the age distribution at diagnosis corroborates other evidence that endometriosis is not unlikely in young women. Data on 'non-text book' symptoms reported by women have been followed up by medical researchers with useful results. The survey is also large enough to allow us to look at rare groups, e.g. therapeutic oophorectomy.

\title{
Emotional Aspects of Endometriosis
}

Lesley Mabbett

Endometriosis Society, London, England

Psychological factors are important in the aetiology and prognosis of endometriosis, as in other conditions, and may be the reason for the marked differences in symptoms among women with a similar extent of disease. $63 \%$ of endometriosis patients sampled suffered from depression and lethargy. This can cause a failure in diagnosis when the endometriosis symptoms are regarded as being psychogenic. A proportion of this depression may be explained by hormone imbalances or thyroid auto-antibodies - detected in a significant percentage of endometriosis cases. However, the experience of frequent, severe or constant pain, infertility and disturbance of marital or other relationships, added to the need to make decisions concerning treatment, conception or surgery, with scant knowledge as to outcome, predispose to anxiety and depression. Information and emotional care are vital. 